\title{
Error Assessment Model for the Inverse Kinematics Problem for Stewart Parallel Mechanisms for Accurate Aerospace Optical Linkage
}

\author{
J. J. Hernández-Gómez $\mathbb{D},{ }^{1}$ I. Medina $\left(\mathbb{D},{ }^{1}\right.$ C. R. Torres-San Miguel $\mathbb{D}$, ${ }^{2}$ \\ A. Solís-Santomé $\mathbb{D},{ }^{1,2}$ C. Couder-Castañeda ${ }^{\mathbb{D}}{ }^{1}$ \\ J. C. Ortiz-Alemán, ${ }^{3}$ and J. I. Grageda-Arellano $\mathbb{D}^{1}$ \\ ${ }^{1}$ Centro de Desarrollo Aeroespacial, Instituto Politécnico Nacional, Mexico \\ ${ }^{2}$ Sección de Estudios de Posgrado e Investigación, Escuela Superior de Ingeniería Mecánica y Eléctrica, Unidad Zacatenco, \\ Instituto Politécnico Nacional, Mexico \\ ${ }^{3}$ Facultad de Ingeniería, Universidad Nacional Autónoma de México, Mexico \\ Correspondence should be addressed to I. Medina; ismesa@gmail.com
}

Received 7 February 2018; Accepted 2 August 2018; Published 3 October 2018

Academic Editor: Alberto Borboni

Copyright (C) 2018 J. J. Hernández-Gómez et al. This is an open access article distributed under the Creative Commons Attribution License, which permits unrestricted use, distribution, and reproduction in any medium, provided the original work is properly cited.

In this work we develop a mathematical model to estimate the error for inverse kinematics problem for Gough-Stewart parallel mechanisms. We propose the estimation error method to include manufacture, assembly, backlash, and sensing errors. We provide the error transmission matrices for the length of each leg of the hexapod, which permits evaluation of the accuracy error in the position of each one, given a desired position and orientation of the mobile platform. We also present numerical modelling in order to estimate the accuracy of the methodology herein proposed, for specific attitude operations corresponding to performing a successful ground-LEO nanosatellite optical link. In such a case, we were able to provide the required tolerances for the actuators in order to guarantee an orientation precision requirement of the order of milliradians.

\section{Introduction}

Space-based laser communications (Lasercom) have the potential to transform scientific, defense, and commercial spacecraft communications platforms. Compared with traditional radio frequency (RF) communications, Lasercom offers higher bandwidth, reduced size and mass of transceivers, lower power consumption, immunity from interference, and higher gain and also avoids the significant regulatory hurdles of radio frequency allocation [1]. Nanosatellites, in particular CubeSats, have great potential to benefit from Lasercom as they are heavily constrained in size, weight, and power [2].

However, the benefits of Lasercom also come with a unique set of challenges. Two of the main problems are the atmospheric loss, since Lasercom frequencies are very susceptible to atmospheric effects, and the pointing, acquisition, and tracking (PAT) subsystem. While narrow transmit beamwidths increase Lasercom link efficiency, the tradeoff is the need for very precise PAT.

Lasercom systems ought to align the optical line-of-sight (LOS) very precisely, with typical systems requiring error as small as submicroradians [3]. Typical pointing accuracies for laser satellite communications (LSC) systems vary from 1 to $200 \mu \mathrm{rad}$; however most typical systems have a pointing accuracy within the range of 10 to $50 \mu \mathrm{rad}$. In the tracking phase, the current values of accuracies typically reach 1 to 3 $\mu \mathrm{rad}[4,5]$.

Lasercom links from low Earth orbit (LEO) to ground present several unique challenges for PAT. The satellite will have to slew rapidly to track the ground station, placing stress on the attitude determination and control subsystem. The fine tracking mechanism to locate a satellite-like spacecraft requires a point-ahead/look-behind calculation, as depicted in Figure 1, considering the distance between Terminal 1 and 


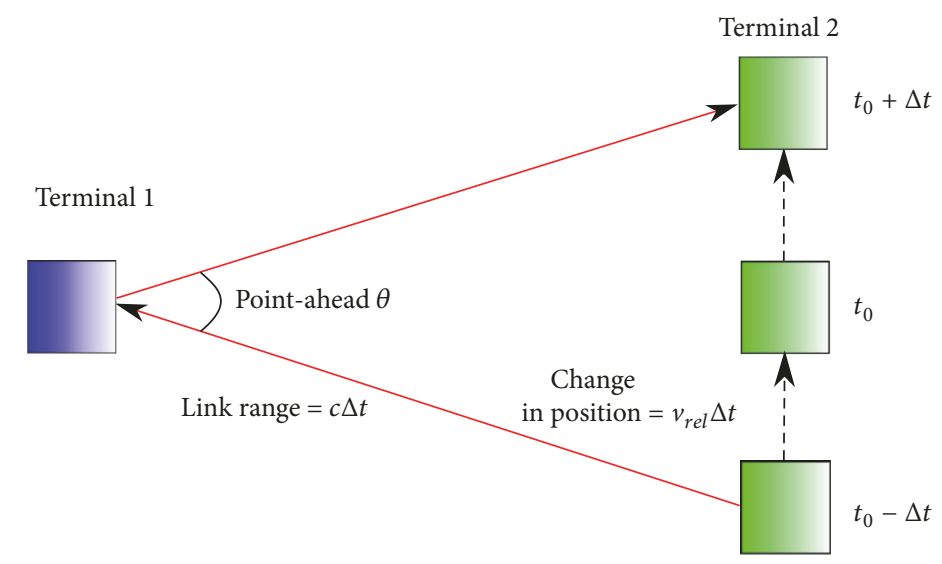

FIGURE 1: Point-ahead/look-behind angle as a function of relative velocity [6].

Terminal 2 at $t_{0}$ as the nominal orbital distance. However, slight variations of the location of Terminal 2 imply the need to consider a point-ahead or look-behind angle $(\theta)$. In order to point at Terminal 2 at $t_{0}$, for instance, the point-ahead ought to be performed at time $t_{0}-\Delta t$, where $\Delta t$ is the time that the link signal takes (travelling at speed of light $c$ ) to travel from Terminal 1 to Terminal 2. Following this principle and the trigonometry from Figure 1, the point-ahead/lookbehind angle would be given by

$$
\theta=2 \arctan \left(\frac{v_{\text {rel }} \Delta t}{c \Delta t}\right) \approx \frac{2 V_{\text {rel }}}{c}
$$

In (1), the last term arises from the fact that the link range is much greater (orbit altitude) than the change in position (Figure 1 does not represent real distances and angles). In this approximation, the point-ahead angle depends only on the relative velocity between the terminals, $V_{\text {rel }}$, and on $c$. Thus, the point-ahead angle given by (1) will be relatively large due to high orbital velocities. Furthermore, ground station sight of view endures less than 10 minutes for LEO satellites, so PAT subsystem ought to quickly acquire and track the ground station to maximise data transmission [6], while keeping great accuracy during the optical link.

Many satellite-to-ground optical communications experiments have been tested by using the optical ground station (OGS) developed by the European Space Agency (ESA) since 2000 [7]. This includes the SILEX project that connected the OGS with the satellite ARTEMIS [8] as well as the SILEX compatible laser communication terminal named LUCE, developed by Japanese Space Exploration Agency (JAXA). The later was successfully tested in 2003 from ESA's optical ground station in a bidirectional space to ground link with ARTEMIS [9]. Other experimental links with TerraSAR-X and NFIRE satellites were tested for only PAT performances [10].

More recently, the laser communication experiment between the satellite SOCRATES and the OGS MeObelonging to the Observatoire de la Côte d'Azur, located in France-has obtained important data from satellite-toground links [11]. JAXA has also been testing ground-tosatellite optical links using the NICT optical ground station in Koganei, Tokyo, since 2006 [12, 13]. Nevertheless, all of these optical aerospace communication experiments have been tested on conventional stationary observatories with two degrees of freedom and limited speed, mostly limiting tests to satellites located in higher orbits.

In order to overcome the problem of atmospheric effects in optical link, we propose the development of a transportable optical ground station. Nevertheless, the mobility of such ground station would increase the requirements of accuracy in its PAT, particularly in the case of ground to LEO links to nanosatellites, due to their high orbital velocity and reduced size. So in order to achieve greater accuracy than conventional PAT systems, we propose to use a GoughStewart platform as PAT at ground station.

Gough-Stewart platform is a parallel mechanism with six degrees of freedom, which is very popular for positioning and high accuracy operations [14-19] because of their great load capacity, as well as their particular high speeds and acceleration, while reducing vibrations. Those characteristics make Gough-Stewart manipulator ideal for optical aerospace linkage. So for a hexapod to provide such accuracy as that required for aerospace optical link from mobile ground station to LEO nanosatellites, the control of the error in the positioning and orientation of the mobile platform in the GoughStewart manipulator is fundamental. Nevertheless, due to the high nonlinearity of its kinematics and dynamics, it is commonly a difficult task to cast theoretical models that accurately describe errors in positioning and orientation of the platform arising, for instance, from manufacturing, assembly, backlash, and sensing. Furthermore, studies focused on determining mathematical models for error in this kind of parallel manipulators are usually casted in an empiric way [20].

Many authors have explored the error and accuracy of parallel mechanisms using different techniques. Altuzarra et al. [21] provide a method to locate regions of the workspace with discontinuities for a planar parallel robot, addressing the error between the pin and the hub of a joint with clearance. Also, stiffness modelling method for overconstrained systems has been used to formulate workspaces as close as possible to a predefined velocity in flexible links [22]. Ding et al. [23] quantify kinematics stiffness error through a simplified method, which combines both analytic and numerical 
modelling (FEA), providing ultrahigh accurate posing applications. Chebbi et al. [24] focus their study on the effects of the clearance in the joints on the parallel robot accuracy, which yields to an analytic predictive model for the pose error. Moreover, Zhang and Gao [25] design kinematic calibration of parallel manipulators assisted though cooperative coevolutionary neural networks (CCNN) to establish a nonlinear relationship between joint variables and the measured pose of the end-effector.

In the case of Gough-Stewart parallel mechanism, some error analysis methods based on differential operators [26] consider errors in the structural dimensions due to the manufacturing and assembling tolerances. Lin et al. [26] demonstrated that such structural errors greatly affect the obtained position of the mobile platform at certain angles. Ropponen and Arai [27] considered the kinematics model taking into account errors in the position of the articulations, as well as action and reaction ones, providing the accuracy in the final positioning of the mobile platform. On the other side, Wang and Ehmann [28] present an error model and accuracy analysis of Stewart platform by focusing on the sources of errors of ball joints, ball joints supports, and motion of ball joints and considering an error model for a oneDOF actuator, while Zhou et al. [29] propose a chain error propagation model based on the differential of $\mathrm{D}-\mathrm{H}$ matrix, where the chains are treated as series single-opened-chains.

Nowadays, each error source can be calculated separately, but up to now there is no theoretical mathematical model able to predict the final position of the legs of the actuator considering all the error sources at the same time. In this work we provide a methodology to calculate the error of position of each leg of the hexapod obtained by considering manufacture, assembly, backlash, and sensing errors, for a desired final position and orientation of the mobile platform with the required accuracy to perform attitude manoeuvres for optical aerospace linkage. This work is organised as follows: In Section 2 we present both the kinematics inverse problem and the development of the error model, where we provide the global error transmission matrix for each leg of the Gough-Stewart mechanism. In Section 3 we provide a parametrisation of the orientation space, and we show a graphical solution of the model herein proposed in order to assess the finest error in actuator's lengths, for given tolerances in manufacturing, assembling, backlash, and sensing, required to satisfy typical accuracy for aerospace optical linkage. Finally, in Section 4 we provide some interesting final remarks of the present work.

\section{Mathematical Modelling of Gough-Stewart Platform}

Parallel manipulators, as Gough-Stewart platform, have many advantages; for example, the payload is shared between the actuators, actuators are located in the base so inertia is reduced, and parallel manipulators exhibit stiff behaviour. On the other hand, they possess some disadvantages as their small workspace, limited dexterity [30], in some cases more energy consumption [31], and a greater sensitivity to input errors [32] as compared to the serial manipulators.

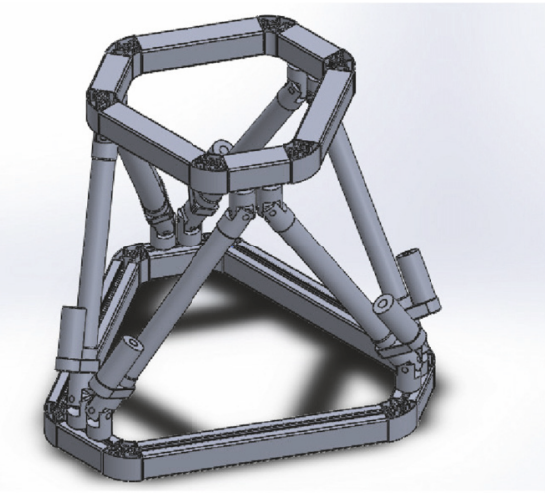

FIGURE 2: 6-6 Gough-Stewart platform.

A problem inherent in Gough-Stewart platform design is that the performance is highly dependent on the geometry; furthermore, different performance measures (workspace, dexterity, etc.) are mutually dependent [33], which complicates the kinematics of the mechanism, exhibiting a highly nonlinear behaviour.

The Gough-Stewart platform consists of 8 main elements that form a hexagonal octahedron, a fixed platform, a mobile platform, and six linear actuators. As shown in Figure 2, the union of the actuators with the platforms is through articulated joints with certain number of degrees of freedom to the fixed platform. Likewise, the other end of each of actuator is connected by another type of gasket to the mobile platform.

The kinematic analysis of the Gough-Stewart platform can be done depending on the input of known variables. Forward modelling consists in the determination of orientation and position of mobile platform, for given lengths of the actuators. The inverse kinematics consists in determining the required lengths on each leg, in order to achieve a certain orientation and position of mobile platform [34, 35]. As in this work we are interested in providing a given accuracy in attitude manoeuvres for aerospace optical link, in what follows we refer only to inverse kinematics of Gough-Stewart platform.

2.1. Inverse Modelling. For Gough-Stewart parallel mechanism, the inverse kinematics consists in determining the length of each leg, given the position and orientation of the mobile platform. It is of great importance when one needs the mobile platform to control its position and orientation, particularly when one needs it to follow a determined trajectory with certain accuracy. In what follows, we shall follow the notation of Gosselin [36].

If the fixed base radium is $R_{B}$ and the angular separation between connection articulations in the base platform is $\phi_{\mathrm{B}}$, then $\vec{b}_{i}$ is the position vector of the articulation of the $i$ th leg from a coordinate system whose origin is attached to the centre of the fixed base (see Figure 3 ) and is given by

$$
\vec{b}_{i}=\left[\begin{array}{c}
R_{B} \cos \theta_{i} \\
R_{B} \sin \theta_{i} \\
0
\end{array}\right], \quad i=1, \ldots, 6,
$$



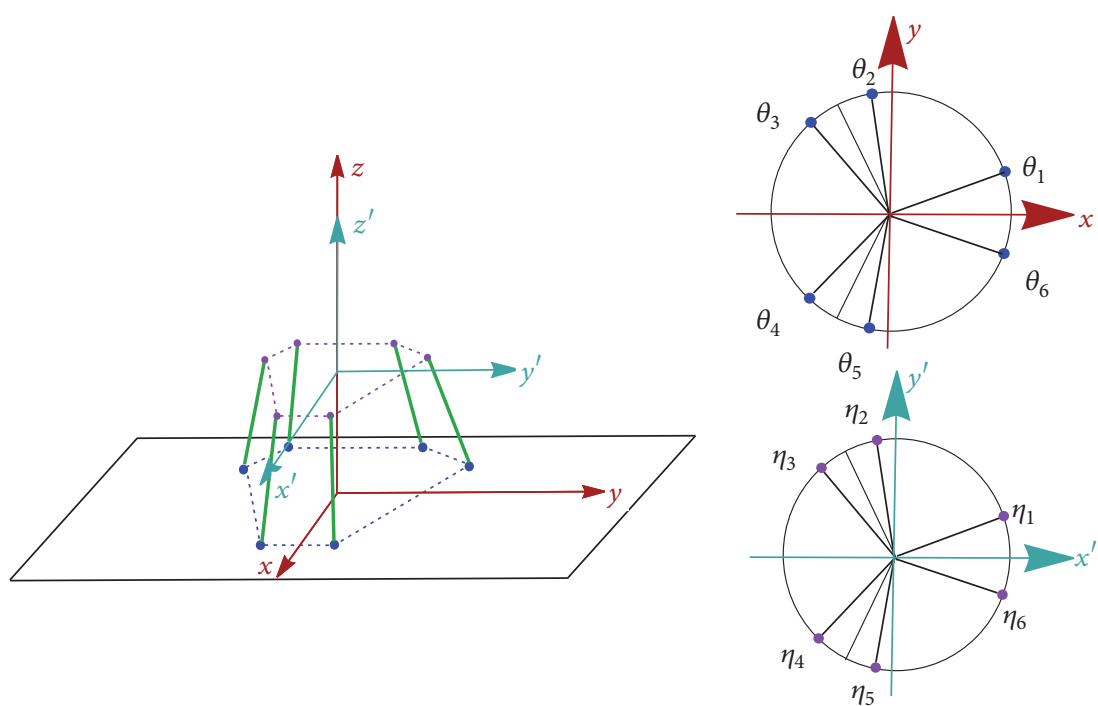

Figure 3: Considered 6-6 Gough-Stewart platform. Base platform in blue, mobile platform in magenta, and actuators in green. Base attached system of reference in red and mobile attached system of reference in cyan.

where $\theta_{i}$ is the angular position of the $i$ th articulation leg in the fixed base and is given by

$$
\left[\begin{array}{l}
\theta_{1} \\
\theta_{2} \\
\theta_{3} \\
\theta_{4} \\
\theta_{5} \\
\theta_{6}
\end{array}\right]=\left[\begin{array}{c}
\phi_{B} \\
\frac{2 \pi}{3}-\phi_{B} \\
\frac{2 \pi}{3}+\phi_{B} \\
\frac{4 \pi}{3}-\phi_{B} \\
\frac{4 \pi}{3}+\phi_{B} \\
-\phi_{B}
\end{array}\right] .
$$

In the same fashion, if the mobile base radium is $R_{p}$ and the angular separation between connection articulations in the mobile platform is $\phi_{\mathrm{p}}$, then $\vec{p}_{i}^{\prime}$ is the position vector of the articulation of the $i$ th leg from a primed coordinate system whose origin is attached to the centre of the mobile base (see Figure 3 ) and is given by

$$
\vec{p}_{i}^{\prime}=\left[\begin{array}{c}
R_{p} \cos \eta_{i} \\
R_{p} \sin \eta_{i} \\
0
\end{array}\right], \quad i=1, \ldots, 6,
$$

where $\eta_{i}$ is the angular position of the $i$ th articulation leg in the mobile base (primed coordinate system) and is given by

$$
\left[\begin{array}{l}
\eta_{1} \\
\eta_{2} \\
\eta_{3} \\
\eta_{4} \\
\eta_{5} \\
\eta_{6}
\end{array}\right]=\left[\begin{array}{c}
\phi_{P} \\
\frac{2 \pi}{3}-\phi_{P} \\
\frac{2 \pi}{3}+\phi_{P} \\
\frac{4 \pi}{3}-\phi_{P} \\
\frac{4 \pi}{3}+\phi_{P} \\
-\phi_{P}
\end{array}\right] .
$$

If the position vector of the mobile platform's centre is given by

$$
\vec{r}=\left[\begin{array}{l}
x \\
y \\
z
\end{array}\right],
$$

measured from the nonprimed coordinate system whose origin lies in the centre of the fixed platform, and if the mobile platform orientation is determined by the rotation matrix

$$
\mathbb{Q}=\left[\begin{array}{lll}
q_{11} & q_{12} & q_{13} \\
q_{21} & q_{22} & q_{23} \\
q_{31} & q_{32} & q_{33}
\end{array}\right],
$$

then the position vector of the articulation in the mobile platform of the $i$ th leg measured from the nonprimed coordinate system is given by

$$
\vec{p}_{i}=\vec{r}+\mathbb{Q}_{i}^{\prime}, \quad i=1, \ldots, 6
$$

Subtracting $\vec{b}_{i}$ from both sides of the later equation yields

$$
\vec{p}_{i}-\vec{b}_{i}=\vec{r}+\vec{Q}_{i}^{\prime}-\vec{b}_{i}
$$

The vector $\vec{p}_{i}-\vec{b}_{i}$ points from the fixed to the mobile articulation of the $i$ th leg, so its length $c_{i}$ is given by its Euclid's norm:

$$
c_{i}=\left\|\vec{p}_{i}-\vec{b}_{i}\right\|=\left\|\vec{r}+\vec{Q}_{\vec{p}_{i}^{\prime}}^{\prime}-\vec{b}_{i}\right\|, \quad i=1, \ldots, 6 .
$$

The general solution to the inverse kinematics problem can be rewritten as

$$
c_{i}=\sqrt{U_{i}^{2}+V_{i}^{2}+W_{i}^{2}}, \quad i=1, \ldots, 6,
$$


where

$$
\begin{aligned}
& U_{i}=x+q_{11} R_{p} \cos \eta_{i}+q_{12} R_{p} \sin \eta_{i}-R_{B} \cos \theta_{i}, \\
& V_{i}=y+q_{21} R_{p} \cos \eta_{i}+q_{22} R_{p} \sin \eta_{i}-R_{B} \sin \theta_{i}
\end{aligned}
$$

and

$$
W_{i}=z+q_{31} R_{p} \cos \eta_{i}+q_{32} R_{p} \sin \eta_{i}
$$

Therefore, for a given (or desired) position of the centre of the mobile platform $\vec{r}$ and orientation $\mathbb{Q}$, the required length of each leg is governed by (11).

2.2. Leg Length Error Modelling. The error in the length of the $i$ th leg, in virtue of (11), is given by

$$
d c_{i}=\frac{1}{c_{i}}\left(U_{i} d U_{i}+V_{i} d V_{i}+W_{i} d W_{i}\right)
$$

where the error in functions $U_{i}, V_{i}$, and $W_{i}$ is given by the following vector relation:

$$
\overrightarrow{d F}_{i}^{T}=\mathscr{M}_{i} \overrightarrow{d E}_{i}^{T}
$$

where $\overrightarrow{d F}_{i}=\left(d U_{i}, d V_{i}, d W_{i}\right), \mathscr{M}_{i}$ is the error transmission matrix for the $i$ th leg, and $\overrightarrow{d E}_{i}$ is a vector that takes into account the global error on each element that determines the length of $i$ th leg. There are thirteen elements per leg that may affect the accuracy of Gough-Stewart platform, so

$$
\begin{aligned}
& \overrightarrow{d E}_{i}=\left(d x, d y, d z, d R_{B}, d R_{P}, d \theta_{i}, d \eta_{i}, d q_{11}, d q_{12}, d q_{21},\right. \\
& \left.\quad d q_{22}, d q_{31}, d q_{32}\right)
\end{aligned}
$$

$d x, d y$, and $d z$ measure the error in position while $d q_{i j} \forall i, j=1, \ldots, 3$, measure the error in attitude determination. The errors in the radii of the base and the platform $\left(d R_{B}\right.$ and $d R_{P}$, respectively), as well as in their angular positions $\left(d \theta_{i}\right.$ and $\left.d \eta_{i} \forall i=1, \ldots, 6\right)$, are the addition of those errors due to manufacture, assembly, backlash, and sensing.

The error transmission matrix for the length of the $i$ th leg is therefore given by

$$
\mathscr{M}_{i}^{T}=\left(\begin{array}{ccc}
1 & 0 & 0 \\
0 & 1 & 0 \\
0 & 0 & 1 \\
-\cos \theta_{i} & -\sin \theta_{i} & 0 \\
q_{11} \cos \eta_{i}+q_{12} \sin \eta_{i} & q_{21} \cos \eta_{i}+q_{22} \sin \eta_{i} & q_{31} \cos \eta_{i}+q_{32} \sin \eta_{i} \\
R_{B} \sin \theta_{i} & R_{B} \cos \theta_{i} & 0 \\
R_{P}\left(q_{12} \cos \eta_{i}-q_{11} \sin \eta_{i}\right) & R_{P}\left(q_{22} \cos \eta_{i}-q_{21} \sin \eta_{i}\right) & R_{P}\left(q_{32} \cos \eta_{i}-q_{31} \sin \eta_{i}\right) \\
R_{P} \cos \eta_{i} & 0 & 0 \\
R_{P} \sin \eta_{i} & 0 & 0 \\
0 & R_{P} \cos \eta_{i} & 0 \\
0 & R_{P} \sin \eta_{i} & 0 \\
0 & 0 & R_{P} \cos \eta_{i} \\
0 & 0 & R_{P} \sin \eta_{i}
\end{array}\right) .
$$

\section{Tolerance Calculations for \\ Given Expected Position and Orientation Errors for Aerospace Optical Link}

For an aerospace optical link, it is necessary to consider the communications laser properties, such as power, wavelength, and divergence angle. In order to achieve a correct position and orientation to establish an accurate link between an optical ground station and a satellite orbiting at LEO orbit (for example, $350 \mathrm{~km}$ of altitude), the laser divergence angle is a critical parameter. The latter actually intervenes in the computation of the required tolerances for the linear actuators of a Gough-Stewart platform. Figure 4 depicts the divergence angle of a laser.
In order to assess the required tolerances of the platform for the specific requirement of orientation precision for PAT at a LEO orbit of $h=350 \mathrm{~km}$ of altitude and considering that the movement of any pointing mechanism is best described by using spherical coordinates, we ought to parametrise rotation matrix (7) in terms of two spherical angles, named $\alpha$ and $\beta$, as follows:

$$
\begin{aligned}
\mathbb{Q} & =\operatorname{Rot}(z ; \alpha) \operatorname{Rot}(x ; \beta) \\
& =\left[\begin{array}{ccc}
\cos (\alpha) & -\cos (\beta) \sin (\alpha) & \sin (\alpha) \sin (\beta) \\
\sin (\alpha) & \cos (\alpha) \cos (\beta) & -\cos (\alpha) \sin (\beta) \\
0 & \sin (\beta) & \cos (\beta)
\end{array}\right],
\end{aligned}
$$




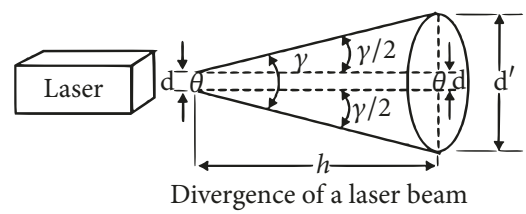

FIGURE 4: Laser beam divergence angle.

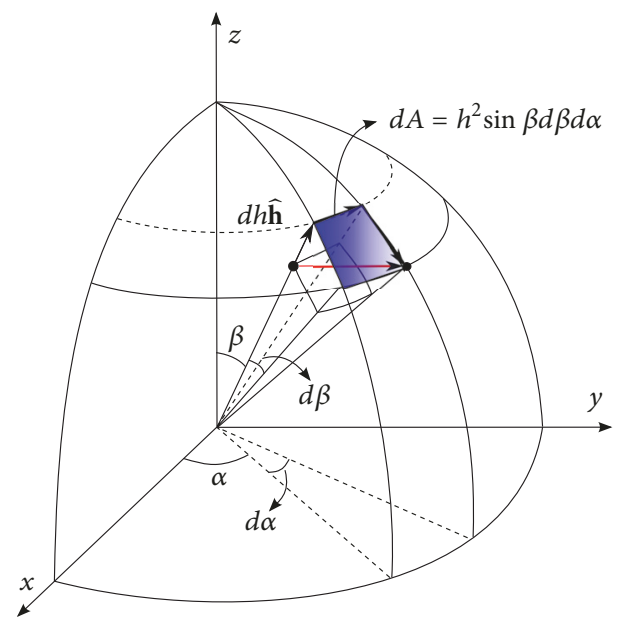

FIgURE 5: Solid angle subtended from PAT to CubeSat.

where $\alpha \in[0,2 \pi)$ and $\beta \in[0, \pi / 2]$, which correspond to the $z$ and $x$ related Euler angles. Thus, the subtended area $d A$ by a solid angle $d \Omega$ from PAT to the CubeSat, since the altitude remains unchanged along all the pointing process, is given by (see Figure 5)

$$
d A=h^{2} d \Omega=h^{2} \sin \beta d \beta d \alpha .
$$

Considering that a satellite has a constant velocity, the accuracy in both $\alpha$ and $\beta$ is the same; i.e., $d \beta=d \alpha$; it is given by

$$
d \beta(\beta)=\sqrt{\frac{d A}{h^{2} \sin \beta}} .
$$

The area $d A$ ought to be estimated as follows: considering that the high power communications laser ray has a divergence angle of $\gamma=4 \mathrm{mrad}$, then

$$
d A=\pi h^{2} \tan ^{2}\left(\frac{\gamma}{2}\right)
$$

is the required area to be matched by the PAT system. Thus, by inserting (22) in (20), the equation for accuracy in $\alpha$ is finally given by

$$
d \beta(\beta)=\sqrt{\frac{\pi \tan ^{2}(\gamma / 2)}{\sin \beta}}=3.545 \mathrm{mrad} .
$$

3.1. Assessment of Required Leg's Tolerances in Positioning. The assessment of the required leg's tolerances $d c_{i}$ for a

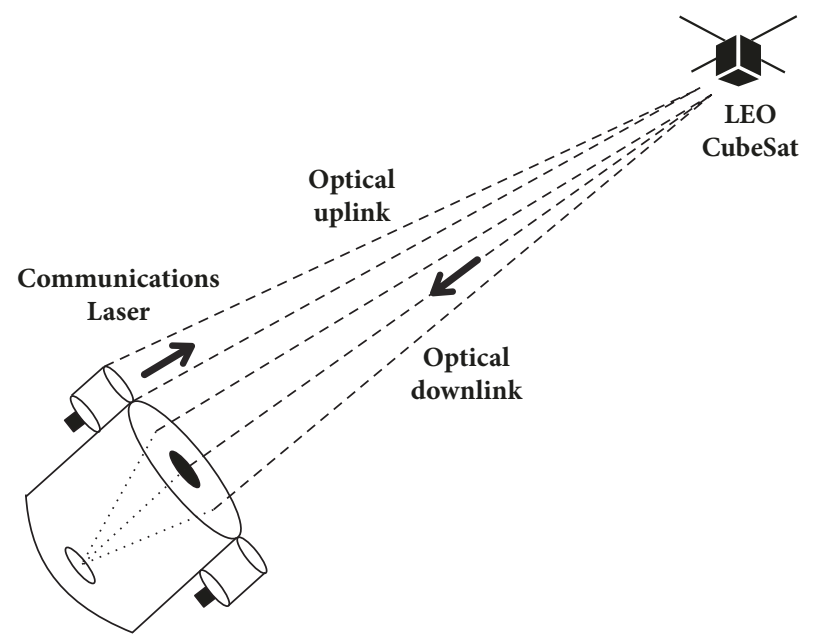

FIGURE 6: Conceptual design of Gough-Stewart platform's payload for optical ground station for LEO satellite link, which consists essentially of a communications laser for the uplink and a telescope for downlink.

specified accuracy in position and orientation for a typical PAT operation, for a given Gough-Stewart platform, can be done as shown in Figure 6.

As the optical ground station (OGS) for LEO satellite link (see Figure 6) consists essentially of a telescope to establish downlink and a laser device for uplink, these elements constitute the payload of Gough-Stewart platform in this work. Considering a $33 \mathrm{~kg}, 10$-inch diameter telescope with a base of 25 inches built into the Gough-Stewart platform with a long exposure adjustment mirror and a fibber to free-space communications laser of $4.23 \times 1.37$ inches embedded to the telescope, the required mobile platform is of $R_{P}=1 \mathrm{~m}$ and $\phi_{P}=\pi / 12 \mathrm{rad}$. Thus, a base platform of $R_{B}=1.5 \mathrm{~m}$ and $\phi_{B}=$ $\pi / 6 \mathrm{rad}$ is optimal to perform the required PAT operations.

This design was achieved by deciding that the (maximal) radius of moving platform $\left(R_{P}\right)$ in order to mount the almost $65 \mathrm{~cm}$ base of the telescope should provide a margin of at least $10 \mathrm{~cm}$ from the base of the telescope to the (minimal radius) edge of the moving platform. This margin is enough for fixing elements of the base of the telescope (without compromising the mechanical integrity of the platform), as well as for placing electronic elements as cables and circuitry for the operation of payload. Then, with considerations of distribution of the weight of payload between the actuators and the constrained workspace (there is a maximum azimuth angle established by ITU for optic links [37], which constrains the workspace of the parallel manipulator), the fixed platform was sized $\left(R_{B}\right)$. The angular design of joints placement $\left(\phi_{P}=\right.$ $\pi / 12 \mathrm{rad}$ and $\left.\phi_{B}=\pi / 6\right)$ responds to the distribution of the weight load between actuators. This joint distribution also guarantees the mobility of passive universal joints in both fixed and moving platforms in the constrained workspace.

Once the platform is specified, errors in the radii of the base and the moving platform, as well as in the angular positions of passive universal joints, due to manufacture, assembly, backlash, and sensing, can be observed in Table 1. 


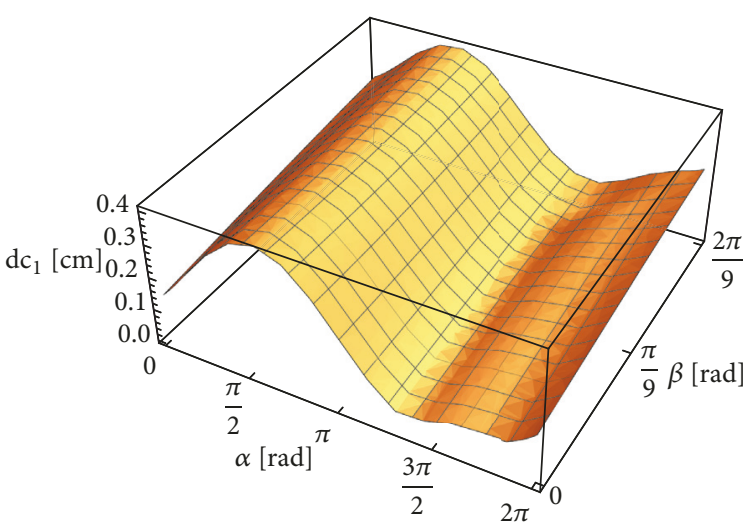

(a) $d c_{1}(\alpha, \beta)$

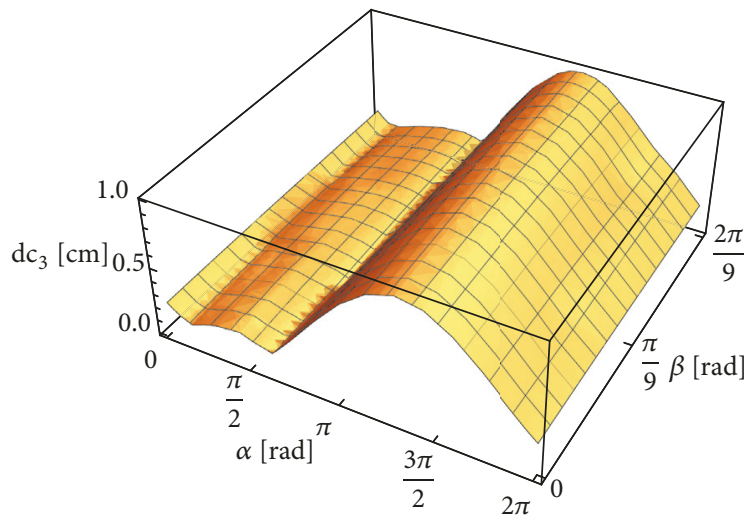

(c) $d c_{3}(\alpha, \beta)$

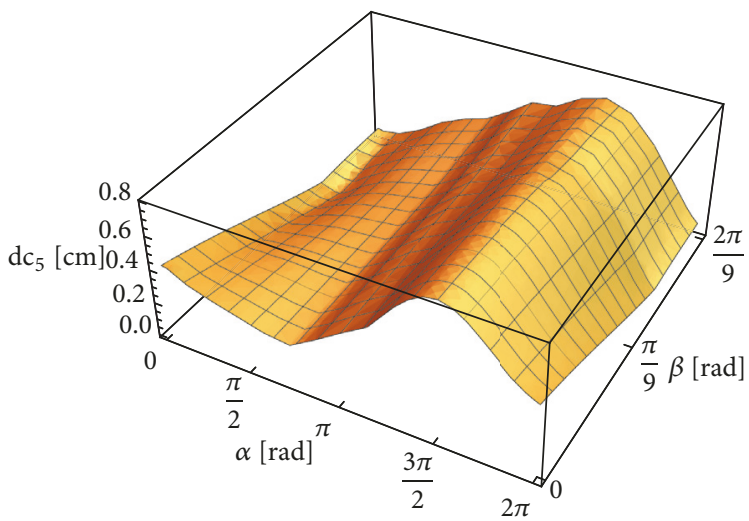

(e) $d c_{5}(\alpha, \beta)$

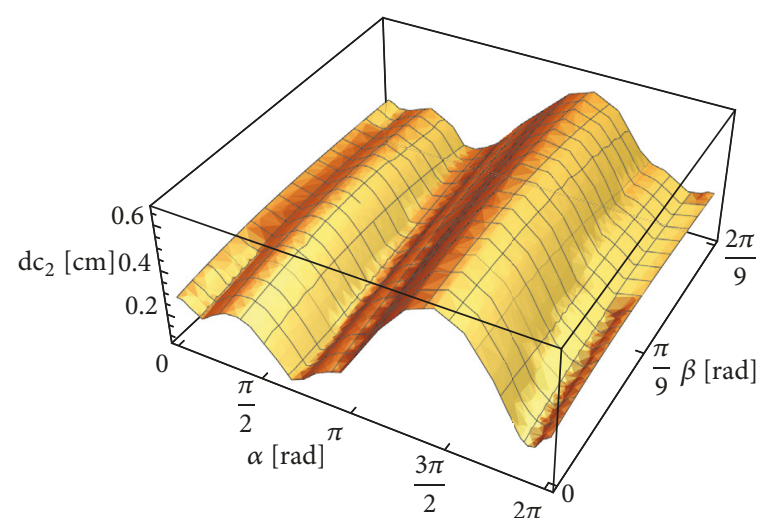

(b) $d c_{2}(\alpha, \beta)$

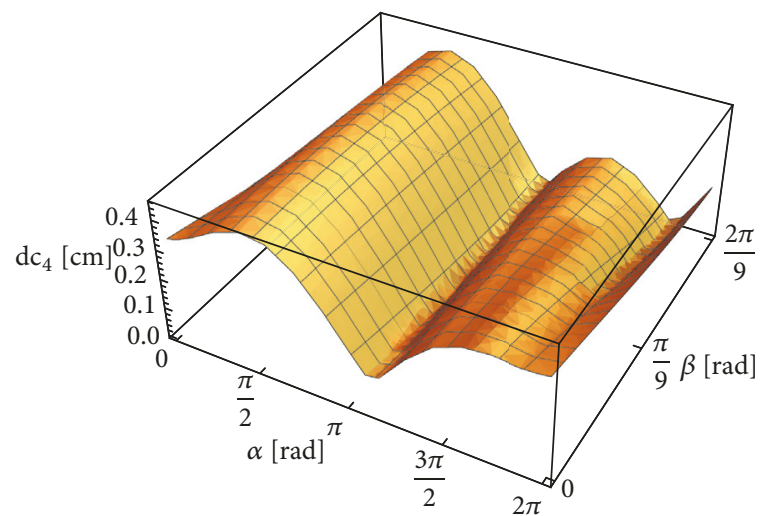

(d) $d c_{4}(\alpha, \beta)$

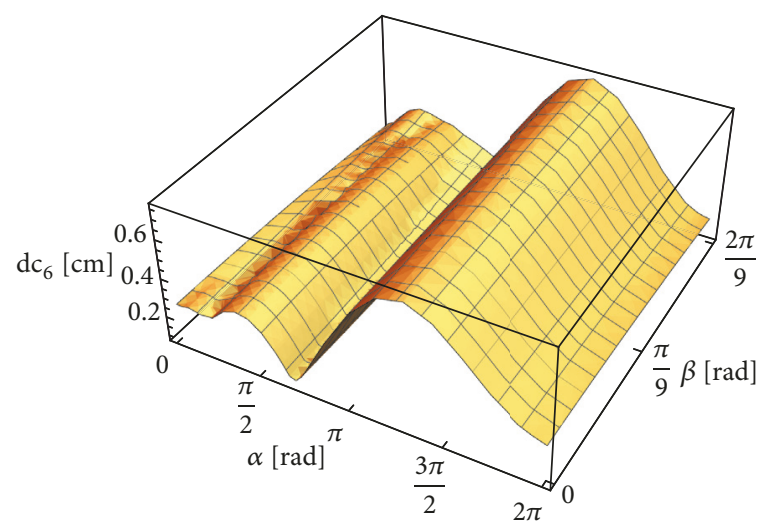

(f) $d c_{6}(\alpha, \beta)$

FiguRE 7: Graphical assessment of the required length error of each leg of the Gough-Stewart platform as function of the orientation angles $\alpha$ and $\beta$. Functions plotted taking the modules of each term in (15).

For this case, we shall consider a position of the mobile platform of $(0,0,45) \mathrm{cm}$, according to (6).

Considering accuracy in orientation given by (23) and errors in Table $1,(15)$ for $i=1, \ldots, 6$ turns into scalar functions of the orientation angles; i.e., $d c_{i}=d c_{i}(\alpha, \beta)$. Thus, it is possible to observe (see Figure 7) in the region of the parameter space $(0 \leq \alpha \leq 2 \pi$ and $0 \leq \beta \leq$ $2 \pi / 9$ (this maximum azimuth angle is the one established by ITU for optic links [37], which constrains the workspace of the parallel manipulator)) the points that require the finest precision in the length of the legs in order to provide the required precision in positioning and orientation. As for the lack of (probably expected) symmetry in the graphs of Figure 7, the scale of the vertical axes of such graphics ought to be taken into account. The depicted changes in $d c_{i} \forall i=$ $1, \ldots, 6$ are very small across the parallel robot workspace, so although important and not negligible they are due to the error configuration of current platform (see Table 1). Furthermore, the distribution of weight along the moving platform is not completely equal; for instance, as mentioned 
TABLE 1: Total errors of the platform due to manufacture, assembly, backlash, and sensing for optical LEO link application.

\begin{tabular}{|c|c|}
\hline Error & Value \\
\hline $\mathrm{d} \theta_{1}(\mathrm{mrad})$ & 1.745 \\
\hline $\mathrm{d} \theta_{2}(\mathrm{mrad})$ & 1.747 \\
\hline $\mathrm{d} \theta_{3}(\mathrm{mrad})$ & 1.744 \\
\hline $\mathrm{d} \theta_{4}(\mathrm{mrad})$ & 1.743 \\
\hline $\mathrm{d} \theta_{5}(\mathrm{mrad})$ & 1.746 \\
\hline $\mathrm{d} \theta_{6}(\mathrm{mrad})$ & 1.745 \\
\hline $\mathrm{d} \eta_{1}(\mathrm{mrad})$ & 1.746 \\
\hline $\mathrm{d} \eta_{2}(\mathrm{mrad})$ & 1.748 \\
\hline $\mathrm{d} \eta_{3}(\mathrm{mrad})$ & 1.743 \\
\hline $\mathrm{d} \eta_{4}(\mathrm{mrad})$ & 1.744 \\
\hline $\mathrm{d} \eta_{5}(\mathrm{mrad})$ & 1.747 \\
\hline $\mathrm{d} \eta_{6}(\mathrm{mrad})$ & 1.745 \\
\hline $\mathrm{dR}_{\mathrm{B}}(\mathrm{mm})$ & 0.15 \\
\hline $\mathrm{dR}_{\mathrm{P}}(\mathrm{mm})$ & 0.1 \\
\hline $\mathrm{dx}(\mathrm{cm})$ & 0.1 \\
\hline dy $(\mathrm{cm})$ & 0.1 \\
\hline $\mathrm{dz}(\mathrm{cm})$ & 0.1 \\
\hline
\end{tabular}

previously, besides the telescope there are other elements as a high power laser device.

Analytically, the precision of each leg can be determined as follows. First of all, critical points ought to be identified for each leg. As $d c_{i}(\alpha, \beta): \mathbb{R}^{2} \longrightarrow \mathbb{R}$ is a scalar function of two variables, the critical points of the $i$ th leg are those in which the first derivative of the function (its gradient) is zero [38]. In other words, these are the set of points $X_{i j}=\left(\alpha_{i j}, \beta_{i j}\right)$ such that

$$
\left.\nabla d c_{i}\right|_{X_{i j}}=0
$$

Then, in order to identify which of those points $\left\{X_{i, j}\right\}$ are the sought global minima, the evaluation of the second derivatives of $d c_{i}$ is required. For a scalar multivariable function, this requires that

$$
\left(H d c_{i}\right)_{X_{i j}}>0
$$

and

$$
\begin{aligned}
\frac{\partial d c_{i}}{\partial \alpha^{2}} & >0 \\
\text { or } \frac{\partial d c_{i}}{\partial \beta^{2}} & >0
\end{aligned}
$$

where $H$ is the determinant of the Hessian matrix of the system [38], formed by all the possible second partial derivatives of the function. For the case of the platform considered in this work, the results for the global minima and their values for each leg are shown in Table 2. From these results, we can observe that the most accurate actuator ought to be leg 5, which ought to possess an error in the length of $d c_{5}= \pm 0.186$ mm.
We ought to remark that the obtained results for each leg (see Table 2) correspond to the orientation which requires most accuracy for it. Also that the reported accuracy for each actuator is different due to the differences in specific errors (Table 1) as well as difference in the distribution of load across the moving platform. On the other side, as we set the accuracy of all actuators as the minimum (of those reported in Table 2), this guarantees that when the ith actuator requires its maximum accuracy, the others are working far from their tolerance. Thus, for instance, if one is to consider actuators with an error of $d c_{i}= \pm 0.15 \mathrm{~mm}$ or $d c_{i}= \pm 0.10 \mathrm{~mm}$, the Gough-Stewart platform would be able to accomplish its pointing within the required orientation error of $d \alpha=d \beta=$ $3.5449124284 \mathrm{mrad}$ (see (23)), with security factors of $19.35 \%$ and $46.24 \%$, respectively.

\section{Conclusions}

In this paper we have developed a mathematical error assessment model for the inverse kinematics for Gough-Stewart parallel mechanisms, which we apply in a case of aerospace optical linkage. Although the model herein developed was intensely derived assuming a 6-6 Gough-Stewart platform, the methodology herein depicted is easily extended to other configurations. Furthermore, the modelling developed in this work has been cast in such a way that we provide transmission error matrices for each leg length, as they are commonly required in the field of instrumentation.

The model, for which we show a graphical and an analytic solutions, has been validated in a scenario with high accuracy requirements in orientation as the establishment of a groundLEO satellite optical link. Although the presented graphical solution heavily relies on posing the error in each leg's lengths as functions of the orientation angles, i.e., $d c_{i}=$ $d c_{i}(\alpha, \beta) \forall i=1, \ldots, 6$, the analytic solution is valid even in cases of spanned solution spaces. For instance, in a more general case, the position of the mobile platform should not be restricted as in the example performed in this paper, yielding a 5-dimensional space in which global mimima ought to be found; i.e., $d c_{i}=d c_{i}(\alpha, \beta, x, y, z) \forall i=1, \ldots, 6$. Moreover, more general expressions for rotation matrix (7) than those used to exemplify the usage of the method herein depicted (see (19)) can be considered, farther increasing the parametric space. Nevertheless, although in such a case no graphical solution is achievable, analytic solution can be equally performed. Furthermore, also numerical assessment of first and second derivatives in order to find global minima of $d c_{i}=d c_{i}(\alpha, \beta, x, y, z) \forall i=1, \ldots, 6$ (see (25) and (26)) can be performed to solve the system. This latter fact shows the generality and validity of the method herein proposed.

\section{Data Availability}

No data were used to support this study.

\section{Conflicts of Interest}

The authors declare that there are no conflicts of interest regarding the publication of this paper. 
TABle 2: Minimum value for the tolerances of each leg, $d c_{i}$, in the orientation domain $\alpha \in[0,2 \pi)$ and $\beta \in[0,2 \pi / 9]$.

\begin{tabular}{lccr}
\hline Leg & Minimum tolerance value & \multicolumn{2}{c}{ Orientation } \\
& $d c_{i}(\mathrm{~cm})$ & $\boldsymbol{\alpha}(\mathrm{rad})$ & $\boldsymbol{\beta}(\mathrm{rad})$ \\
\hline $\mathbf{1}$ & 0.1416 & 0.0000 & 0.0000 \\
$\mathbf{2}$ & 0.1977 & 0.3829 & 0.0000 \\
$\mathbf{3}$ & 0.1217 & 0.0000 & 0.6979 \\
$\mathbf{4}$ & 0.1794 & 0.2940 & 0.6898 \\
$\mathbf{5}$ & 0.0186 & 0.0531 & 0.4846 \\
$\mathbf{6}$ & 0.1982 & 0.6981 \\
\hline
\end{tabular}

\section{Acknowledgments}

The authors acknowledge EDI grant by SIP/IPN. This research was partially supported by SIP/IPN Projects 20181139, 20180472, 20181441, 20181028, 20181141, and 1931.

\section{References}

[1] J. Císar, O. Wilfert, F. Fanjul-Vélez, N. Ortega-Quijano, and J. L. Arce-Diego, "New trends in laser satellite communications: design and limitations," in Photonics, Devices, and Systems IV, p. 71382I, International Society for Optics and Photonics, 2008.

[2] A. Mehrparvar, "CubeSat design specification, revision 13," Tech. Rep., California Polytechnic State University, 2015.

[3] R. Marshalek, "Pointing, Acquisition, and Tracking," in NearEarth Laser Communications, vol. 20094921 of Optical Science and Engineering, CRC Press, 2009.

[4] C. H. Chalfant III, F. J. Orlando, J. Gregory et al., "Novel laser communications transceiver with internal gimbal-less pointing and tracking," in International Symposium on Optical Science and Technology, pp. 194-201, 2002.

[5] B. L. Wilkerson, D. Giggenbach, and B. Epple, "Concepts for fast acquisition in optical communications systems," in In Proceedings of spie the international society for optical engineering, vol. 6304, International Society for Optical Engineering, USA, August 2006.

[6] K. M. Riesing, Development of a pointing, acquisition, and tracking system for a nanosatellite laser communications module [Ph.D. thesis], Massachusetts Institute of Technology, 2015.

[7] S. Alluru, A System Design of an Optical Wireless Communication System for CubeSats [Ph.D. thesis], University of Florida, 2010.

[8] M. R. Garcia-Talavera, S. Chueca, A. Alonso, T. Viera, and Z. Sodnik, "Analysis of the preliminary optical links between ARTEMIS and the Optical Ground Station," in Proceedings of the International Symposium on Optical Science and Technology, pp. 33-43, International Society for Optics and Photonics, Seattle, WA.

[9] J. Romba, J. C. Ricklin, D. G. Voelz et al., "ESA's bidirectional space-to-ground laser communication experiments," in Proceedings of the Optical Science and Technology, the SPIE 49th Annual Meeting, pp. 287-289, International Society for Optics and Photonics, 2004.

[10] Z. Sodnik, A. K. Majumdar, C. C. Davis, J. Perdigues Armengol, R. H. Czichy, and R. Meyer, "Adaptive optics and ESA's optical ground station," in SPIE Optical Engineering+ Applications, p. 746406, International Society for Optics and Photonics.

[11] G. Artaud, J.-L. Issler, N. Védrenne et al. et al., "Laser communication experiments between Sota and MEO optical ground station," in Proceedings of the international Conference on Space Optics-ICSO '16, vol. 10562, p. 105624B, Biarritz, France, October 2017.

[12] M. Toyoshima, K. Takizawa, T. Kuri et al., "An overview of the KODEN experiment between the OICETS satellite and the optical ground station in NICT," Elektrotechnik und Informationstechnik, vol. 124, no. 6, pp. 193-199, 2007.

[13] M. Toyoshima, T. Sasaki, H. Takenaka et al., "Research and development of free-space laser communications and quantum key distribution technologies at NICT,' in Proceedings of the International Conference on Space Optical Systems and Applications, ICSOS '11, pp. 1-7, USA, May 2011.

[14] K. Furutani, M. Suzuki, and R. Kudoh, "Nanometre-cutting machine using a Stewart-platform parallel mechanism," Measurement Science and Technology, vol. 15, no. 2, pp. 467-474, 2004.

[15] C. Hirt, G. Papp, A. Pál, J. Benedek, and E. Szũcs, "Expected accuracy of tilt measurements on a novel hexapod-based digital zenith camera system: A Monte-Carlo simulation study," Measurement Science and Technology, vol. 25, no. 8, 2014.

[16] G. Hu, X. Li, and X. Yan, "Inverse kinematics model's parameter simulation for stewart platform design of driving simulator," Lecture Notes in Electrical Engineering, vol. 419, pp. 887-898, 2018.

[17] Z. Li, X. Cao, D. Wang, W. Yang, and B. Zhang, "Optimization of Calibration Method for the Secondary Mirror Fine Tuning Stewart Mechanism of Optoelectronic Telescope," Bandaoti Guangdian/Semiconductor Optoelectronics, vol. 38, no. 3, pp. 451-458, 2017.

[18] X. Yang, H. Wu, Y. Li, and B. Chen, "Dynamic isotropic design and decentralized active control of a six-axis vibration isolator via Stewart platform," Mechanism and Machine Theory, vol. 117, pp. 244-252, 2017.

[19] A. Dabiri, S. Sabet, M. Poursina, D. G. Armstrong, and P. E. Nikravesh, "An optimal Stewart platform for lower extremity robotic rehabilitation," in Proceedings of the 2017 American Control Conference, ACC 2017, pp. 5294-5299, USA, May 2017.

[20] E. Hernez, S. I. Valdez, and E. Sanchez, "On the numerical modelling and error compensation for general gough-stewart platform," International Journal of Advanced Robotic Systems, vol. 11, 2014.

[21] O. Altuzarra, J. Aginaga, A. Hernández, and I. Zabalza, "Workspace analysis of positioning discontinuities due to clearances in parallel manipulators," Mechanism and Machine Theory, vol. 46, no. 5, pp. 577-592, 2011.

[22] A. Pashkevich, D. Chablat, and P. Wenger, "Stiffness analysis of overconstrained parallel manipulators," Mechanism and Machine Theory, vol. 44, no. 5, pp. 966-982, 2009. 
[23] B. Ding, B. S. Cazzolato, R. M. Stanley, S. Grainger, and J. J. Costi, "Stiffness analysis and control of a stewart platformbased manipulator with decoupled sensor-actuator locations for ultrahigh accuracy positioning under large external loads," Journal of Dynamic Systems, Measurement, and Control, vol. 136, no. 6, 2014.

[24] A.-H. Chebbi, Z. Affi, and L. Romdhane, "Prediction of the pose errors produced by joints clearance for a 3-UPU parallel robot," Mechanism and Machine Theory, vol. 44, no. 9, pp. 1768-1783, 2009.

[25] D. Zhang and Z. Gao, "Optimal kinematic calibration of parallel manipulators with pseudoerror theory and cooperative coevolutionary network," IEEE Transactions on Industrial Electronics, vol. 59, no. 8, pp. 3221-3231, 2012.

[26] H. Lin, C. Meng, and H. Chuan, "Error analysis of parallel manipulators based on differential operators," in Proceedings of the IEEE International Conference on Mechatronics and Automation, ICMA '09, pp. 1302-1306, China, August 2009.

[27] T. Ropponen and T. Arai, "Accuracy analysis of a modified Stewart platform manipulator," in Proceedings of the 1995 IEEE International Conference on Robotics and Automation. Part 1 (of 3), pp. 521-525, May 1995.

[28] S.-M. Wang and K. F. Ehmann, "Error model and accuracy analysis of a six-DOF Stewart Platform," Journal of Manufacturing Science and Engineering, vol. 124, no. 2, pp. 286-295, 2002.

[29] X. Zhou, F. Zhou, and Y. Wang, "Pose error modeling and analysis for 6-DOF stewart platform," in Proceedings of the 2017 Chinese Automation Congress (CAC), pp. 6470-6475, Jinan, October 2017.

[30] M. Ceccarelli, Fundamentals of mechanics of robotic manipulation, vol. 27, Springer Science Business Media, 2004.

[31] M. Morisawa, T. Yakoh, T. Murakami, and K. Ohnishi, "A comparison study between parallel and serial linked structures in biped robot system," in Proceedings of the 26th Annual Confierence of the IEEE (Industrial Electronics Society, 2000), vol. 4, pp. 2614-2619, IECON, 2000.

[32] S. Briot and I. A. Bonev, "Are parallel robots more accurate than serial robots?" Transactions of the Canadian Society for Mechanical Engineering, vol. 31, no. 4, pp. 445-455, 2007.

[33] G. Wu, "Multiobjective optimum design of a 3-RRR spherical parallel manipulator with kinematic and dynamic dexterities," Modeling, Identification and Control, vol. 33, no. 3, pp. 111-122, 2012.

[34] W. Zhou, W. Chen, H. Liu, and X. Li, "A new forward kinematic algorithm for a general Stewart platform," Mechanism and Machine Theory, vol. 87, pp. 177-190, 2015.

[35] R. V. Petrescu, R. Aversa, A. Apicella et al., "Inverse Kinematics of a Stewart Platform," Journal of Mechatronics and Robotics, vol. 2, no. 1, pp. 45-59, 2018.

[36] C. Gosselin, Kinematic analysis, optimization and programming of parallel robotic manipulators [Ph.D. thesis], Université de Sherbrooke, 1988.

[37] ITU, “Technical and operational characteristics of satellites operating in the range 20-375 THz," in Proceedings of the Technical report, International Telecommunications Union. RECOMMENDATION ITU-R S.1590, 2002.

[38] M. Spivak, Calculus On Manifolds: A Modern Approach To Classical Theorems Of Advanced Calculus, CRC Press, 2018. 


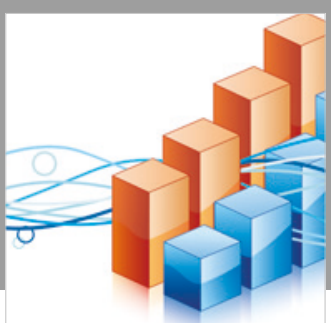

Advances in

Operations Research

\section{-n-m}
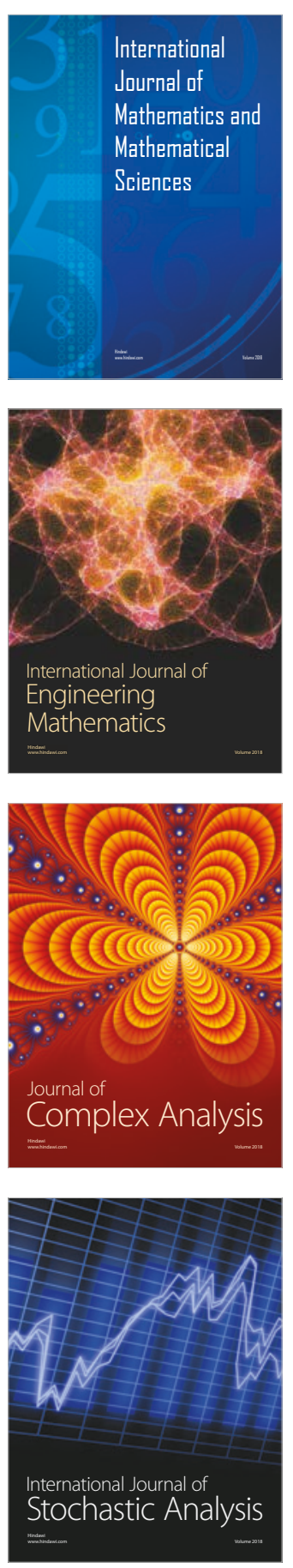
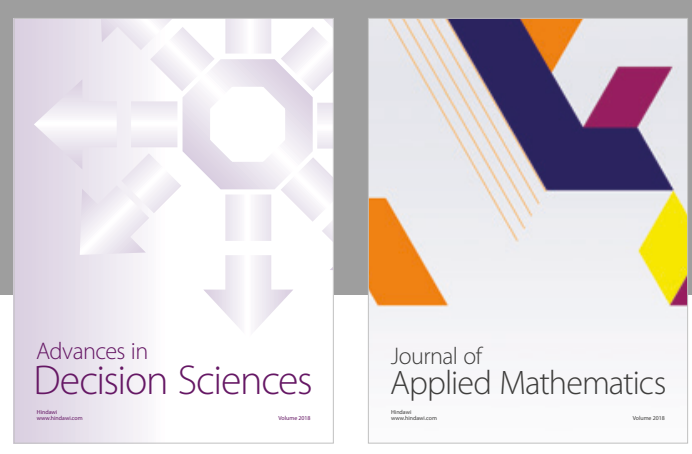

Journal of

Applied Mathematics
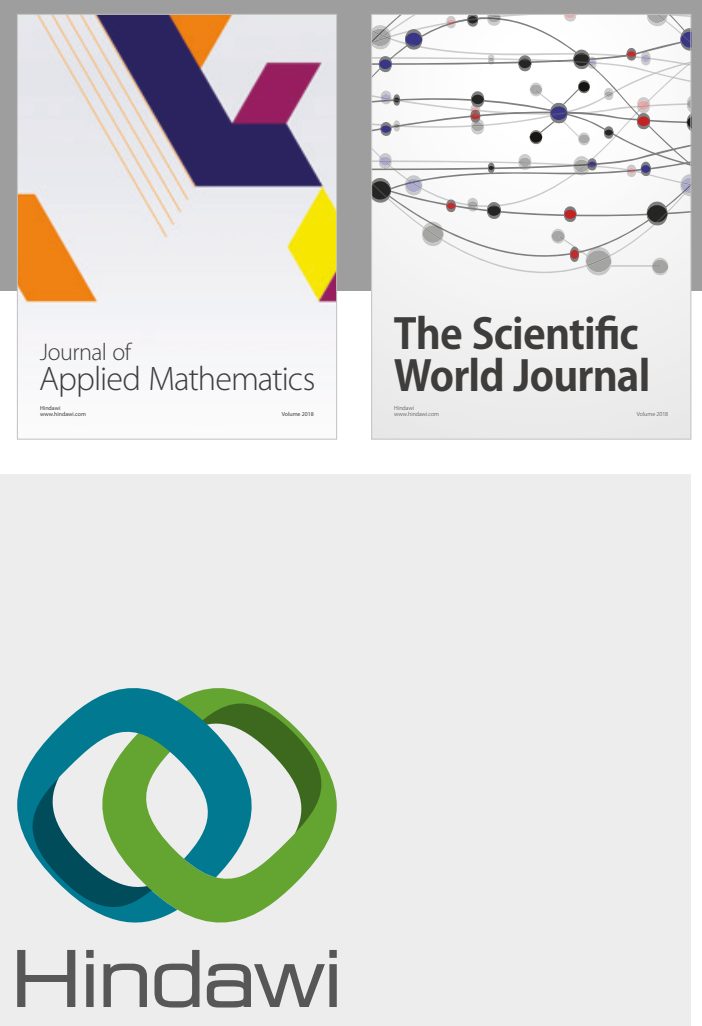

Submit your manuscripts at

www.hindawi.com

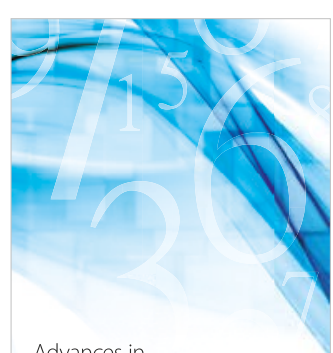

Advances in
Numerical Analysis
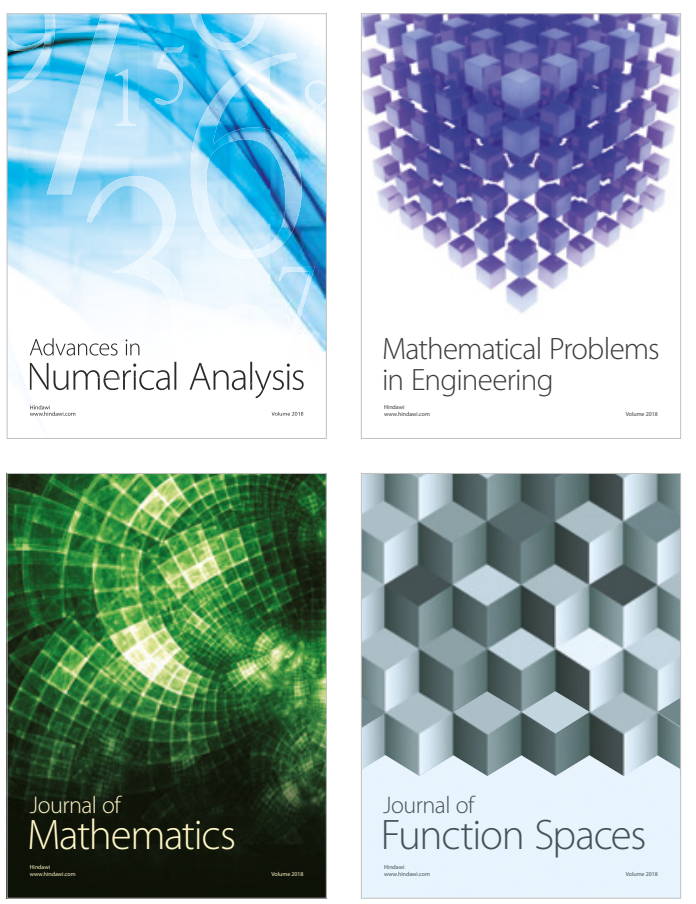

Mathematical Problems in Engineering

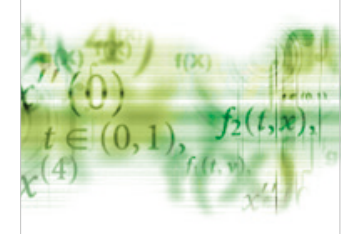

International Journal of

Differential Equations

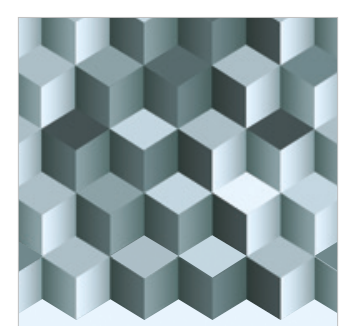

Journal of

Function Spaces

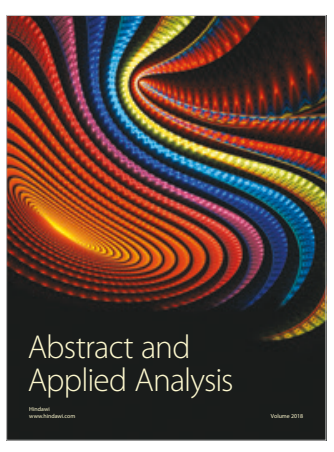

The Scientific

World Journal

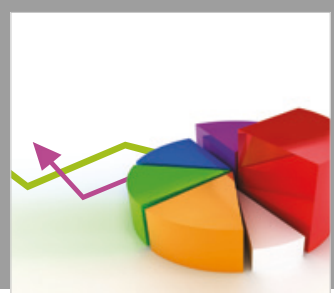

Journal of

Probability and Statistics
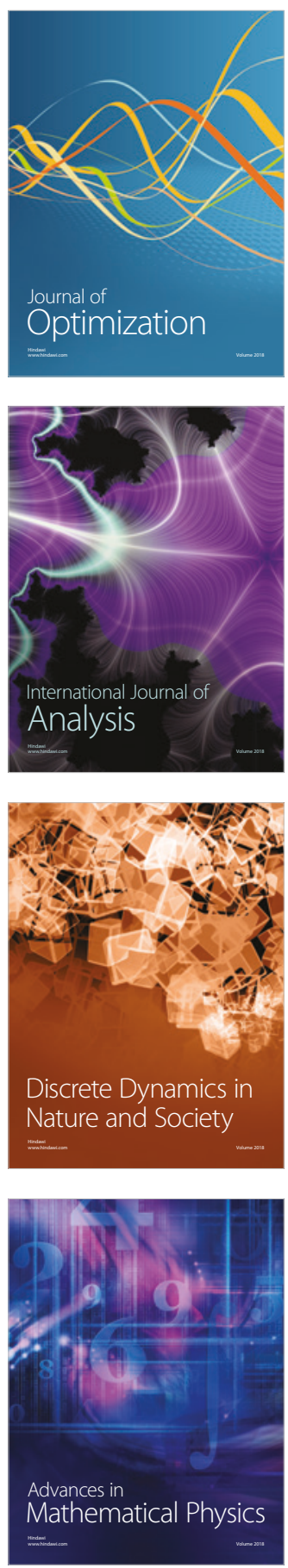